\title{
Characterization of In-Vivo Proton Magnetic Resonance Spectroscopic Patterns of Intracranial Space-Occupying Lesions: A 1-Year Observational Study
}

\author{
Rajendra V. Mali ${ }^{1}$, Aseem Katyal ${ }^{1}$ \\ ${ }^{1}$ Department of Radiology, Jawaharlal Nehru Medical College, Nehru Nagar KLE University, Belagavi 590010, Karnataka, India
}

Corresponding author: Dr Rajendra V. Mali, Department of Radiology, Jawaharlal Nehru Medical College, Nehru Nagar KLE University, Belagavi 590010, Karnataka, India

DOI: http://dx.doi.org/10.21276/ijcmsr.2018.3.4.29

How to cite this article: Rajendra V. Mali, Aseem Katyal. Characterization of in-vivo proton magnetic resonance spectroscopic patterns of intracranial space-occupying lesions: a 1-year observational study. International Journal of Contemporary Medicine Surgery and Radiology. 2018;3(4):D126-D130.

\section{A B S T R A C T}

Introduction: Magnetic resonance (MR) spectroscopy is a new and emerging imaging modality, which offers a detailed biochemical analysis of the tissues thereby giving us a glimpse of in-vivo human brain metabolism. This study was undertaken to assess the role of MR spectroscopy in characterization and diagnosis of intracranial space-occupying lesions (ICSOLs) when used in addition to conventional magnetic resonance imaging (MRI) and to distinguish between neoplastic and nonneoplastic lesions.

Material and Methods: This 1-year observational study was conducted at the Department of Radiodiagnosis, KLE'S Hospital, Belgaum. A total of 50 patients with ICSOLs detected by MRI brain (plain/contrast) scans from January 2012 to December 2012 were studied.

Results: Majority of the patients were women (56\%) and the most common age-group was 31-45 years. Headache and neurological deficits were the most frequent presenting complaints. Out of total cases, $64 \%$ of the lesions were neoplastic and $36 \%$ of the lesions were non-neoplastic. High choline (Cho)/creatine $(\mathrm{Cr}$ ) and Cho/ $\mathrm{N}$-acetyl aspartic acid (NAA) ratios were found in neoplastic lesions as compared to non-neoplastic lesions. Among the neoplastic lesions, high-grade gliomas had significantly higher $\mathrm{Cho} / \mathrm{Cr}$ and $\mathrm{Cho/NAA}$ ratios as compared to low-grade gliomas. Lactate and lipid resonances were also present in high-grade gliomas. Among the infectious lesions, abscesses had amino acid peaks and high lactate levels; however, they had low Cho/Cr andCho/NAA ratios, which helped to differentiate them from neoplasms. Tuberculomas demonstrated a prominent lipid peak.

Conclusion: Information provided by MR spectroscopy complemented the information obtained by MRI and was particularly helpful in distinguishing neoplastic from non-neoplastic lesions.

Key words: Intracranial Space-Occupying lesion; Magnetic Resonance Imaging; Magnetic Resonance Spectroscopy; Neoplastic Lesions; Non-Neoplastic Lesions

\section{INTRODUCTION}

Any newly altered, malignant, or non-malignant, primary or metastatic neoplasms including parasitic, as well as infectious lesions, residing inside the cranium is termed as intracranial space-occupying lesions (ICSOL). ${ }^{1}$ Tumors that initiate from the brain or neural cells are known as primary intracranial tumors, which are more complex and significant in number than secondary intracranial tumors that originate outside. Among all primary neoplasms of whole human body, primary space-occupying intracranial neoplasms account for $9 \%$ and of all intracranial neoplasms they account for 40-50\%. In elderly population, cases of brain tumor are increasing $^{2}$ and approximately 80,000 to 100,000 new cases of secondary brain tumors are diagnosed every year. ${ }^{2}$ So far, only two risk factors are found convincing to cause brain tumor that are ionizing radiations and inherited mutations of penetrant genes. ${ }^{3}$ Reports suggest that intracranial neoplasms configuration and occurrence considerably vary with age, sex, tribal, as well as geographical region. ${ }^{4-6}$ ICSOLs also include non-neoplastic lesions such as cerebral infarcts, intracerebral hematomas, brain abscess. ${ }^{7}$ So priority during diagnosis relies on whether the lesions are neoplastic or nonneoplastic, which helps in accurate differential diagnosis for proper management of patients with ICSOL. ${ }^{8}$ Computed tomography (CT) and magnetic resonance imaging (MRI) are the widely used primary stage imaging modalities for the diagnosis of patients suspected with brain lesions. ${ }^{9}$ Although MRI is significant in diagnosis; however, in some cases, in differentiation of tumoral mass from neoplastic to nonneoplastic lesions, it remains incompetent even after contrast administration. ${ }^{8}$ In some of the cases, exact differentiation becomes intricate and not possible. Thus, patients have to undergo invasive procedures to rule out malignant lesions. 
Magnetic resonance spectroscopy (MRS) is a device employed to determine the presence and molecular structure of compounds. It can deliver the biochemical, as well as metabolic information of the brain that assists in noninvasive differential diagnosis. ${ }^{10} \mathrm{MRS}$ renders images based on the metabolites in the tissue. ${ }^{9}$ Major metabolites that can be detected by MRS are choline (Cho), creatine $(\mathrm{Cr})$, $\mathrm{N}$-acetyl aspartate (NAA), lactate, myoinositol, glutamine lipids etc. MRS also plays a significant clinical role in observing the evolution of diseases such as stroke, ischemic injury, dementia, acquired immunodeficiency syndrome (AIDS), and intracranial neoplasms. ${ }^{10}$ Latest improvements in the technique also provide additional information on energy uptake, necrotic specifics of the cells, and proliferation or degradation of the cells. ${ }^{11}$ Combination of this MRS with MRI showed a promising results in previous reports for distinguishing neoplasms from non-neoplastic pathology. ${ }^{12-14}$ However, further research and clinical trials are needed to address above issues, and in India, due to lack of availability of MRS, less studies have been reported. Hence, the current study is intended to assess the role of MRS in characterization and diagnosis of ICSOLs when used in addition to conventional MRI and to distinguish between neoplastic and non-neoplastic lesions.

\section{MATERIAL AND METHODS}

This 1-year observational study was conducted at KLES Dr. Prabhakar Kore Hospital and Medical Research Centre, Belgaum from January 2012 to December 2012. A total of 50 patients with ICSOLs detected by MRI of the brain (plain or contrast) scans were selected for the study. Patients with traumatic lesions, cerebrovascular accidents, arteriovenous malformations, calcifications, benign cystic lesions, and patients not willing to participate in the study were excluded from the study. Ethical clearance was obtained from institutional ethics committee and selected patients were informed about the nature of the study and a written informed consent was obtained. Study population was interviewed for demographic data such as age, sex, and clinical history; and clinical examination were noted.

\section{Imaging}

A 1.5 T MRI manufactured by SIEMENS with software version SYNGO 2002 B was used for scanning and field of view (FOV) was set to $14-24 \mathrm{~cm}$. Slice thickness was fixed to 10,5 , and $3 \mathrm{~mm}$ and matrix size to $256 \times 256$. Plain images were acquired and injected with gadolinium diethylene triaminepentaacetic acid (DTPA) at a dose of $0.1 \mathrm{mmol} / \mathrm{kg}$ body weight. Subsequent to injection, contrast images were acquired and assessed. MRI of brain was performed in supine position and proper positioning and immobilization of head was obtained. Standard head coil was used for acquisition of images. Precontrast scanning was done using diffusionweighed images (DWI), T1, T2, and PD. Multihance was used as contrast agent in dose of $0.1 \mathrm{mmol} / \mathrm{kg}$ body weight. Postcontrast coronal, sagittal, and axial spin-echo T1weighed images (T1W1) were obtained. If required, thinner sections were obtained in the region of interest wherever necessary and if lesions were suggestive of a tumor in MRI, then were subjected to proton $\left({ }^{1} \mathrm{H}\right)$ MRS examination.

\section{${ }^{1} \mathrm{H}$ Magnetic resonance spectroscopy}

Global shimming and local shimming were carried out before spectroscopic measurements to adjust for static and dynamic magnetic field inhomogeneties. The global shimming was optimized at $15-17 \mathrm{~Hz}$, and full width at half maximum (FWHM) value was set between 5-7 Hz. A gaussian pulse was used for water suppression. Areas of edema, adjoining calvarium, ventricles, and paranasal sinuses were avoided to prevent signal contamination. Optimal shimming and water suppression were achieved in most cases; however, the location and heterogeneous nature of the lesions prevented optimal shimming in some patients (eight cases) and such patients were excluded from the study. ${ }^{1} \mathrm{H}$ MRS was carried out using single- (SV) and multivoxel (MV) techniques at short echo time (TE; $30 \mathrm{~ms})$, long TE (270 ms), and intermediate $\mathrm{TE}(135 \mathrm{~ms})$ wherever appropriate with an acquisition time of approximately 3-6 min each using stimulated echo acquisition method (STEAM) sequence. The voxel size was selected depending upon the size of the lesion and whether single voxel or multivoxel MRS is being used. Based on the literature, prior experience with normal subjects and software standardization values of $\mathrm{Cho} / \mathrm{Cr}$ $>1.5, \mathrm{NAA} / \mathrm{Cr}<1.6$, and $\mathrm{Cho} / \mathrm{NAA}>0.8$ were taken as abnormal. Standard of reporting was verified and confirmed by experienced radiologists who worked in consultation and had access to all available clinical and imaging information. The images were analyzed for number of lesions, tumor size, location, signal intensity changes, areas of hemorrhage, cystic changes, calcification, and intensity and pattern of contrast enhancement. MRS results were evaluated for the distribution of pathologic spectra across the lesion and for signal ratios in different lesions. The metabolites and ratios assessed were: NAA)/Cr), Cho/Cr, Cho/NAA; lactate, lipids and other prominent peaks in the spectrum.

\section{STATISTICAL ANALYSIS}

The data obtained werecoded and entered into Microsoft Excel Worksheet (Annexure IV). The categorical data were expressed as rates, ratios, proportions, and percentages. The continuous data were expressed as mean \pm standard deviation and the comparison was done using two sample ' $\mathrm{t}$ ' test. A probability value ( $P$ value) of less than or equal to 0.050 was considered as statistically significant.

\section{RESULTS}

In the present observational study, amongst the cases with ICSOLs women preponderance was observed (56\%); and $44 \%$ were men. Leading age-group was found between 31 and 45 years (34\%) and followed by $46-60$ years (30\%) age-group. Most common presentations were headache and neurological deficits (44\%) followed by seizures (40\%). A total of 31 patients had single lesion while, 19 had multiple lesions with metastases, infection, or multiple sclerosis.

T1-weighted MRI sequence revealed hypointense lesions in $54 \%$ of the patients and $24 \%$ of patients had isointense lesions. T2-weighted MRI sequence revealed hyperintense on $50 \%$ of patients and $40 \%$ had mixed intense lesions. In the present study, on contrast administration, there was intense enhancement in 54\% of patients, moderate in 30\%, and mild 


\begin{tabular}{|l|c|c|c|c|c|c|}
\hline \multirow{2}{*}{ Lesions } & \multicolumn{2}{|c|}{ Choline to creatine } & \multicolumn{2}{c|}{ Choline to N-acetyl aspartate } & \multicolumn{2}{c|}{ N-acetyl aspartate to creatine } \\
\cline { 2 - 7 } & Mean & SD & Mean & SD & Mean & SD \\
\hline High-grade gliomas & 4.58 & 1.07 & 4.98 & 0.30 & 0.92 & 0.22 \\
\hline Low-grade gliomas & 1.88 & 0.51 & 1.85 & 0.77 & 1.07 & 0.26 \\
\hline Lymphomas & 3.70 & 1.27 & 1.85 & 0.07 & 1.95 & 0.78 \\
\hline Metastasis & 3.50 & 1.32 & 2.96 & 0.90 & 1.18 & 0.38 \\
\hline Meningiomas & 6.90 & 1.96 & 7.38 & 3.10 & 0.98 & 0.19 \\
\hline Infections & 1.47 & 0.36 & 1.43 & 0.46 & 1.06 & 0.26 \\
\hline Others (MS) & 1.40 & 0.30 & 1.38 & 0.56 & 1.00 & 0.43 \\
\hline \multicolumn{7}{|r|}{} \\
\hline
\end{tabular}

\begin{tabular}{|c|c|c|c|c|c|c|}
\hline \multicolumn{7}{|c|}{ Mean metabolite ratio in neoplastic and nonneoplastic lesions } \\
\hline \multirow[t]{2}{*}{ Lesions } & \multicolumn{2}{|c|}{ Choline to creatine } & \multicolumn{2}{|c|}{ Choline to $\mathrm{N}$-acetyl aspartate } & \multicolumn{2}{|c|}{$\mathrm{N}$-acetyl aspartate to creatine } \\
\hline & Mean & SD & Mean & SD & Mean & SD \\
\hline Neoplastic & 4.10 & 1.98 & 4.07 & 2.35 & 1.03 & 0.39 \\
\hline Non-neoplastic & 1.45 & 0.34 & 1.41 & 0.45 & 1.05 & 0.28 \\
\hline$P$ value & \multicolumn{2}{|c|}{$<0.001$} & \multicolumn{2}{|c|}{$<0.001$} & \multicolumn{2}{|c|}{0.826} \\
\hline \multicolumn{7}{|c|}{ Mean metabolite ratio in High-grade gliomas and Low-grade gliomas } \\
\hline High-grade gliomas & 4.57 & 1.07 & 4.98 & 0.30 & 0.91 & 0.21 \\
\hline Low-grade gliomas & 1.88 & 0.51 & 1.85 & 0.76 & 1.06 & 0.25 \\
\hline$P$ value & \multicolumn{2}{|c|}{$<0.001$} & \multicolumn{2}{|c|}{$<0.001$} & \multicolumn{2}{|c|}{0.272} \\
\hline
\end{tabular}

\begin{tabular}{|l|c|c|c|c|}
\hline Lesions & MRI + MRS & MRI Alone & $\begin{array}{c}\text { two or more } \\
\text { differential diagnosis }\end{array}$ & Positive correlation (\%) \\
\hline Neoplasms & 32 & 35 & 3 & $(90.62)$ \\
\hline Intraaxial & 21 & 24 & 3 & $21(85.71)$ \\
\hline High-grade gliomas & 8 & 11 & 1 & $8(62.50)$ \\
\hline Low-grade gliomas & 6 & 5 & 1 & $5(83.33)$ \\
\hline Metastasis & 5 & 6 & 0 & $5(83.33)$ \\
\hline Lymphoma & 2 & 2 & 0 & $(100)$ \\
\hline Extraaxial & 11 & 11 & 0 & $11(100)$ \\
\hline Meningioma & 6 & 6 & 0 & $6(100)$ \\
\hline Others & 5 & 5 & 7 & $5(100 \%)$ \\
\hline Infections & 15 & 22 & 0 & $15(53.33)$ \\
\hline Tuberculoma & 8 & 11 & 4 & $8(62.5)$ \\
\hline Neurocysticercosis & 3 & 3 & 0 & $3(100)$ \\
\hline Abscess & 3 & 7 & 0 & $3(57.14)$ \\
\hline Others & 1 & 3 & 1 & $1(100)$ \\
\hline Others & 3 & & $3(100)$ \\
\hline \multicolumn{2}{|c|}{ Table-3: Correlation of Magnetic resonance imaging } & Magnetic resonance spectroscopy with Magnetic resonance imaging alone \\
\hline
\end{tabular}

enhancement in $12 \%$ of patients. However, two patients did not show any contrast enhancement.

In the present study, the MRI enhancement pattern was found to be peripheral in 50\% (mostly in high-grade gliomas, abscesses, and granulomatous lesions) of cases, heterogeneous in $22 \%$ of cases, homogenous in $18 \%$ (mostly in meningiomas), and patchy in $6 \%$ of cases. Also, $64 \%$ of the cases had neoplastic lesions and among these, intraaxial neoplasms were present in $65 \%$ and extraaxial in $35 \%$ of cases. Infectious etiology was seen in $30 \%$ of cases and tuberculomas were the predominant infectious lesions in $53 \%$ of infectious lesions. Inflammatory tumefactive lesions such as multiple sclerosis were noted in $6 \%$ of cases.

The mean $\mathrm{Cho} / \mathrm{Cr}$ and $\mathrm{Cho} / \mathrm{NAA}$ ratios were elevated in the neoplastic lesions as compared to infections and multiple sclerosis and were highest among meningiomas and high grade gliomas, while NAA/Cr was found to be inconclusive and did not follow any set pattern among neoplastic and non-neoplastic lesions. However, 75\% of high-grade gliomas and metastases cases had lactate. Lipids were seen in $62 \%$ of high-grade glioma cases and $40 \%$ of metastases cases. In meningiomas, $40 \%$ of cases had alanine, which was not seen in any other tumor cases.

In neoplastic lesions, high grade gliomas clearly had higher $\mathrm{Cho} / \mathrm{Cr}$ and $\mathrm{Cho} / \mathrm{NAA}$ ratios when compared to low-grade gliomas, infectious lesions, as well as nonneoplastic lesions. However, NAA/Cr metabolite ratio was found to be non significant. (Tables 1-2)

MRS allowed better characterization of lesions and helped to narrow down the multiple differential diagnosis given by 

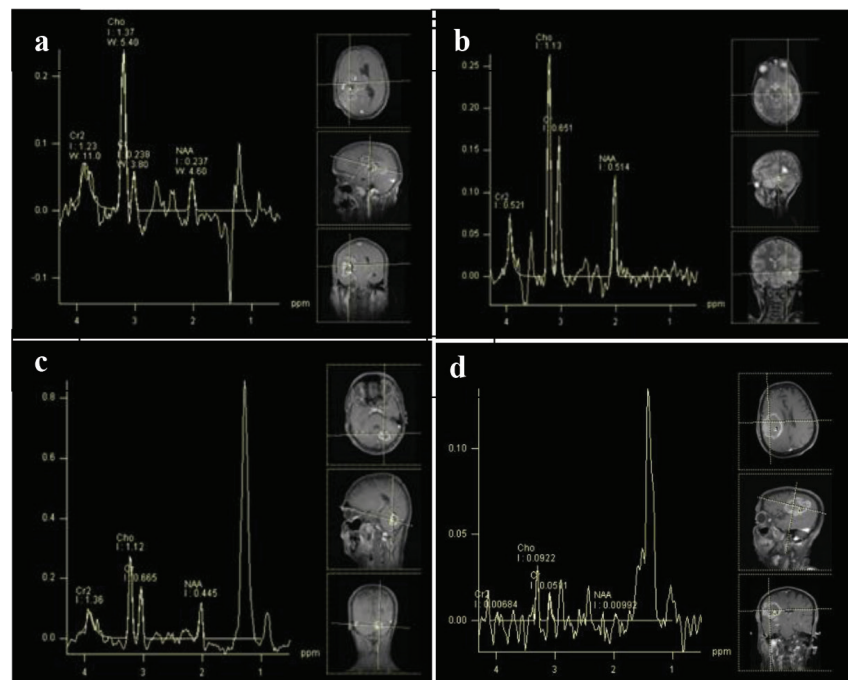

Figure-1: MR spectroscopy (MV STEAM, TE: 135): (a) glioblastoma multiforme, (b) low grade glioma, (c) tuberculoma, (d) cerebral abscess

MRI, particularly in case of differentiating non-neoplastic lesions such as abscesses from high grade tumors (Table 3; Figure.1).

\section{DISCUSSION}

MRI has established superiority over $\mathrm{CT}^{15,16}$ and has become an important diagnostic tool in evaluation of ICSOLs. Multiplanar acquisition and high-contrast resolution between different tissues have made MRI very sensitive in tumor detection and localization. Certain neoplasms such as malignant glioblastoma multiforme, metastasis tumors; and certain non-neoplastic lesions such as abscess or acute infarction show contrast enhancement on MRI. However, low-grade gliomas and non-neoplastic diseases such as cortical dysplasia and contusion are usually non-enhancing. ${ }^{11}$ This uncertainty in contrast enhancement of neoplastic and nonneoplastic lesions and lack in its specificity limits the use of MRI. ${ }^{9}$ Exact categorization of cerebral lesions requires the estimation of connection between the pathological process as well as their metabolic profile. ${ }^{9}{ }^{1} \mathrm{H}-\mathrm{MRS}$ is an emerging non-invasive imaging modality, which offers a detailed biochemical examination of tissues providing a glimpse of in-vivo human brain metabolism. It renders five principal metabolite peaks such as choline comprising compounds peaks representing membrane turnover, creatinine peak replicating energy synthesis, NAA peaks serving as a marker of neuronal cells, lactate representing anaerobic metabolism, and lipid reflects dead necrotic cells. ${ }^{17}$ MRS characterizes neoplasms by elevated Choline and lowered NAA levels signifying that the Choline peak acts as a biomarker. ${ }^{11}$

In the present study, $56 \%$ of patients were women and $44 \%$ were men. Mean age of patients presenting ICSOL were between 30 and 45 years. Present study found neoplasms (64\%) as the predominant cause of ICSOLs. Irrespective of causes, predominant symptoms were headache and neurological deficits followed by seizures, which is in concordance with the study done by Bhavesh et al. ${ }^{18}$ where chief presentation was headache followed by seizures. It also has been reported that brain tumors and headache are correlated clinically ${ }^{19}$ and seizures occurs in $95 \%$ of patients presenting with brain tumor. ${ }^{20}$ Infections, either tuberculomas or abscesses, did not present with any fever or meningismus making diagnosis difficult clinically. These observations were in concordance with the findings of Grand et al. ${ }^{21}$

All neoplasms revealed elevated Cho, and decreased NAA and creatinine levels, which manifest the presence of carcinoma characteristics in majority of the patients. Mild elevation of Cho level in infectious lesions may be due to membrane degradation products. Among neoplasms, the mean Cho/ $\mathrm{Cr}$ and Cho/NAA ratiosweresignificantly elevated in eightpatients with high grade gliomas as compared to lowgrade gliomas in sixcases. High ratios indicated increased grade of malignancy. So it is concluding to the certain studies that suggested $\mathrm{Cho} / \mathrm{Cr}$ and $\mathrm{Cho} / \mathrm{NAA}$ ratios can act as a biomarker for determining carcinoma characteristics in the brain tissues as well as for grading of malignancies. ${ }^{11,22,23}$ $\mathrm{NAA} / \mathrm{Cr}$ levels in both low- and high-grade gliomas werefound to be nonsignificant, which is having same conclusion as in study done by Sutton et al. ${ }^{23}$ Neuronal marker-NAA levels are relatively less in extraaxial ones when compared to intraaxial ones as extraaxials lacks neural tissues; however, intraaxial possess neural tissue. However, their levels depend on difference in the cellular composition and the degree of necrosis. ${ }^{24}$

It has been postulated that presence of lipid signals may be useful in grading gliomas. ${ }^{25}$ In the present study none of the low-grade gliomas showed presence of lipids, while it was present in $62 \%$ of cases of high-grade gliomas. This concludes that lipid resonances indicates necrosis, and presence of lipids correlates with higher degrees of malignancy. In meningiomas, alanine resonances are seen. ${ }^{24}$ In our study, alanine peaks were found in two of six patients and none of the other ICSOLs demonstrated alanine peaks. Including our study, Cho et $\mathrm{al}^{26}$ showed schwannomas cases with elevated Choline and myoinositol levels and decreased NAA levels. Out of two patients, one patient showed a MI peak whereas in the second patient MI was not seen. This could be due to reason that the second patient underwent spectroscopy at intermediate TE.

Abscesses are difficult to differentiate from cystic or necrotic neoplasms in MRI because both of them show peripheral ring enhancement patterns. ${ }^{27,28}$ However, a high $\mathrm{Cho} / \mathrm{Cr}$ and Cho/NAA levels were seen in high-grade neoplasms and amino acids resonances (two out of three patients) were seen only in abscesses. This is in concordance with Harada et $\mathrm{al}^{29}$ and concludes the statement that the MR spectra of amino acids are not seen in brain tumors cases. ${ }^{28}$

In our study (six out of eight cases), tuberculous lesions demonstrated lipid resonances, which were not seen in other infective lesions except for one case of abscess. Lipid peaks were absent in neurocysticercosis, which had similar imaging appearance on MRI. Low Cho/NAA levels helped to differentiate tuberculomas from other high-grade neoplastic lesions, which showed similar pattern of ring enhancement. This suggests combination of MRS not only compliments the information available from MRI but also helps in assessing the prognosis and response to treatment. Present study has shown that MRS can differentiate between neoplastic and 
nonneoplastic ICSOLs and help in diagnosing various lesions based on the metabolite spectrum and ratios.

\section{REFERENCE}

1. ZAB J. Pattern of intra-cranial space occupying lesions: experience at King Khalid University Hospital. Ann Saudi Med 1989;9(1):3-10.

2. Edward R. Laws KT. Brain Tumors. CA Cancer J Clin. 1993;43 (3):263-71.

3. Schwartzbaum JA, Fisher JL, Aldape KD, Wrensch M. Epidemiology and molecular pathology of glioma. Nat Clin Pract Neurol. 2006;2 (5):494-503

4. Wrensch M, Minn Y, Chew T, Bondy M, Berger MS. Epidemiology of primary brain tumors: current concepts and review of the literature. Neuro Oncol. 2002;4 (4):278-99.

5. M. Ejaz Butt Sak, Naseer A. Chaudrhy, G. R. Qureshi. Intra-cranial space occupying lesions a morphological analysis Biomedica. 2005;21 (2):31-5.

6. Wrensch M, Bondy ML, Wiencke J, Yost M. Environmental risk factors for primary malignant brain tumors: a review. J Neurooncol. 1993;17:47-64.

7. Overton MC, 3rd, Haynie TP, Snodgrass SR. Brain Scans in Nonneoplastic Intracranial Lesions: Scanning with Chlormerodrin $\mathrm{Hg} 203$ and Chlormerodrin $\mathrm{Hg}$ 197. JAMA. 1965;191 (6):431-6.

8. Majos C, Aguilera C, Alonso J, Julia-Sape M, Castaner S, Sanchez JJ, et al. Proton MR spectroscopy improves discrimination between tumor and pseudotumoral lesion in solid brain masses. AJNR Am J Neuroradiol. 2009;30 (5):544-51.

9. Evanthia Kousi ITaKE. Proton Magnetic Resonance Spectroscopy of the Central Nervous System Novel Frontiers of Advanced Neuroimaging 2013.

10. Cousins JP. Clinical MR spectroscopy: fundamentals, current applications, and future potential. AJR Am J Roentgenol. 1995;164 (3):1337-47.

11. Nagar VA, Ye J, Xu M, Ng WH, Yeo TT, Ong PL, et al. Multivoxel MR spectroscopic imaging--distinguishing intracranial tumours from non-neoplastic disease. Ann Acad Med Singapore. 2007;36 (4):309-13.

12. Cianfoni A, Niku S, Imbesi SG. Metabolite findings in tumefactive demyelinating lesions utilizing short echo time proton magnetic resonance spectroscopy. AJNR Am J Neuroradiol. 2007;28 (1):272-7.

13. Mishra AM, Gupta RK, Jaggi RS, Reddy JS, Jha DK, Husain N, et al. Role of diffusion-weighted imaging and in vivo proton magnetic resonance spectroscopy in the differential diagnosis of ring-enhancing intracranial cystic mass lesions. J Comput Assist Tomogr. 2004;28 (1):540-7.

14. Kumar A, Kaushik S, Tripathi RP, Kaur P, Khushu S. Role of in vivo proton MR spectroscopy in the evaluation of adult brain lesions: our preliminary experience. Neurol India. 2003;51 (3):474-8.

15. Smits M. Imaging of oligodendroglioma. Br J Radiol. 2016;89 (2):20150857.

16. Schmidt GP, Kramer H, Reiser MF, Glaser C. Wholebody magnetic resonance imaging and positron emission tomography-computed tomography in oncology. Top Magn Reson Imaging. 2007;18 (5):193-202.
17. Borja MJ, Plaza MJ, Altman N, Saigal G. Conventional and advanced MRI features of pediatric intracranial tumors: supratentorial tumors. AJR Am J Roentgenol. 2013;200 (6):W483-503.

18. Bhavesh R Goyani BVU, Parth Naik, Hinal Bhagat, Mahesh K Vadel, Rakesh Sheth. A Study on Role OF Magnetic Resonance Imaging (MRI) in Intracranial Space Occupying Lesions NJMR. 2015;5 (7):18-21.

19. Kirby S, Purdy RA. Headaches and brain tumors. Neurol Clin. 2014;32 (1):423-32.

20. DeAngelis LM. Brain Tumors. New England Journal of Medicine. 2001;344 (6):114-23.

21. Grand S, Lai ES, Esteve F, Rubin C, Hoffmann D, Remy C, et al. In vivo $1 \mathrm{H}$ MRS of brain abscesses versus necrotic brain tumors. Neurology. 1996;47 (5):846-8.

22. Fountas KN, Karampelas I. Proton magnetic resonance spectroscopic imaging can predict length of survival in patients with supratentorial gliomas. Neurosurgery. 2004;55 (4):257-8; author reply 8.

23. Sutton LN, Wang ZJ, Wehrli SL, Marwaha S, Molloy P, Phillips PC, et al. Proton spectroscopy of suprasellar tumors in pediatric patients. Neurosurgery. 1997;41 (1):388-94; discussion 94-5.

24. Kugel H, Heindel W, Ernestus RI, Bunke J, du Mesnil R, Friedmann G. Human brain tumors: spectral patterns detected with localized H-1 MR spectroscopy. Radiology. 1992;183 (4):701-9.

25. Poptani H, Gupta RK, Roy R, Pandey R, Jain VK, Chhabra DK. Characterization of intracranial mass lesions with in vivo proton MR spectroscopy. AJNR Am J Neuroradiol. 1995;16 (1):1593-603.

26. Cho YD, Choi GH, Lee SP, Kim JK. (1)H-MRS metabolic patterns for distinguishing between meningiomas and other brain tumors. Magn Reson Imaging. 2003;21 (3):663-72.

27. Garg RK, Sinha MK. Multiple ring-enhancing lesions of the brain. J Postgrad Med. 2010;56:307-16.

28. Lai PH, Ho JT, Chen WL, Hsu SS, Wang JS, Pan $\mathrm{HB}$, et al. Brain abscess and necrotic brain tumor: discrimination with proton MR spectroscopy and diffusion-weighted imaging. AJNR Am J Neuroradiol. 2002;23 (6):1369-77.

29. Harada M, Tanouchi M, Miyoshi H, Nishitani H, Kannuki S. Brain abscess observed bylocalized proton magnetic resonance spectroscopy. Magn Reson Imaging. 1994;12 (1):1269-74.

\section{Source of Support: Nil; Conflict of Interest: None}

Submitted: 11-10-2018; Accepted: 20-12-2018; Published online: 31-12-2018 